\title{
Ausubel's principle of prior knowledge in first year mechanics
}

\section{David Sands \\ Department of Physical \\ Sciences \\ University of Hull \\ Hull \\ HU6 7RX}

d.sands@hull.ac.uk

\section{In Physics education research has revealed that students can demonstrate alternative conceptions of the physical world that are not only stubbornly resistant to change but can actively inhibit the learning of Newtonian ideas.}

\begin{abstract}
The Force Concept Inventory, a 30-question multiple choice test, has been used to test the baseline knowledge in mechanics prior to a course of instruction at Hull over the three years corresponding to entry in 2008, 2009 and 2010. Students whose pre-university education occurred outside the UK or who were repeating the year have been excluded from the analysis in order to focus attention on first-time UK students. These constitute the great majority of the entrants and the results essentially characterise the entry-level knowledge of a typical cohort. Two interesting findings have emerged. First, there is a wide range of abilities within each cohort, as judged by the test scores, and secondly, analysis of the scores question by question reveals a remarkable consistency between the different cohorts. This consistency extends even to the distribution of choices within individual questions. Five such questions are analysed in detailed to reveal which aspects of mechanics a typical class finds difficult. Ausubel's principle of first finding out what students know in order to teach accordingly can therefore be applied not to the individual students but to the class as a whole and suggestions as to how instruction might be tailored to address the weaknesses revealed by the Force Concept Inventory are discussed.
\end{abstract}

\section{Introduction}

It was David Ausubel who famously wrote", "The most important single factor influencing learning is what the learner already knows. Ascertain this and teach him accordingly". Nowhere is this more true than in mechanics. Physics education research has revealed that students can demonstrate alternative conceptions of the physical world that are not only stubbornly resistant to change but can actively inhibit the learning of Newtonian ideas. For example, Andrea di Sessa ${ }^{2}$ reports in a study from the 1980 s that both graduate students and young children exhibit very similar naïve views, implying that these alternative views develop early in childhood and can persist right through the subsequent years of formal education, even beyond graduation. It's not enough simply to determine that students don't know, say, Newton's third law of motion, we also need to know what view they hold in its stead. Fortunately, this is relatively easy in mechanics as the force concept inventory $(\mathrm{FCl})$ provides a well known test of understanding in mechanics ${ }^{3}$. Indeed, the $\mathrm{FCl}$ has played some part in revealing how common are some of these alternative conceptions.

For readers not familiar with the $\mathrm{FCl}$, and who might in consequence regard it as simply a questionnaire about mechanics, some background about its development is necessary. The questionnaire was developed over many years following interviews with students about their views of mechanics and is designed to test not only whether students are familiar with, and can use, Newtonian concepts, but also what concepts might be held instead. The possible answers to the questions incorporate the naïve views of mechanics concepts revealed by the interviews, such as the so-called "impetus principle" that force must exist in the direction of motion. The idea behind this is to avoid the principal weakness of multiple choice questionnaires, namely that respondents might simply guess the answers. The premise upon which the $\mathrm{FCl}$ is based is that students either know the answer or believe they know the answer and therefore have no need to guess. A few questions also require qualitative reasoning in order to arrive at the answer and the question then tests the application of Newtonian concepts. Following the development of the $\mathrm{FCl}$ concept inventories are now finding application in a range of disciplines outside physics ${ }^{4}$, but the $\mathrm{FCl}$ is still the most widely used. 
The validity of the $\mathrm{FCl}$ as an instrument for measuring conceptual understanding is much discussed within the open literature ${ }^{4,5,6}$. In particular, the question has arisen ${ }^{7,8,9}$ as to whether the $\mathrm{FCl}$ provides a measure of a student's coherent understanding of the force concept or whether it provides a snapshot of different aspects of their knowledge and understanding. This question is side-stepped here by focussing explicitly on the responses to specific questions. For the past three years the first year cohort at Hull has been tested using the $\mathrm{FCl}$ prior to a course of instruction in mechanics based around modelling in VPython ${ }^{10}$. The pattern of responses is examined question by question to show how the $\mathrm{FCl}$ reveals the collective knowledge, misunderstandings and deficiencies among typical entrants to a UK physics degree. Five questions in particular are selected to illustrate problems with understanding Newton's third law of motion and the existence of alternative conceptions such as the impetus principle.

\section{Methodology}

The $\mathrm{FCl}$ was given to the majority of the class prior to instruction in mechanics in order to establish their baseline knowledge. The intention was to test all students in order to determine the baseline knowledge of the class prior to instruction, but those who were repeating the year or whose pre-university education occurred outside the UK were excluded from this analysis in order to concentrate primarily on the knowledge of typical UK university entrants. Ideally the whole class would have been tested, but some students were absent and were not subsequently tested. Nonetheless, a large majority of the eligible students from each cohort was tested; $84.8 \%$ in $2008,96.0 \%$ in 2009 and $75.4 \%$ in 2010 . The mechanics course was run in semester two for each of the years represented in this survey and in 2008 and 2009 the test was administered during the first class. In 2010 the test was administered during the induction week following registration, and this difference appears to be responsible for the reduction in the number of students tested. The tests were all untimed, with students being left to complete the test in their own time.

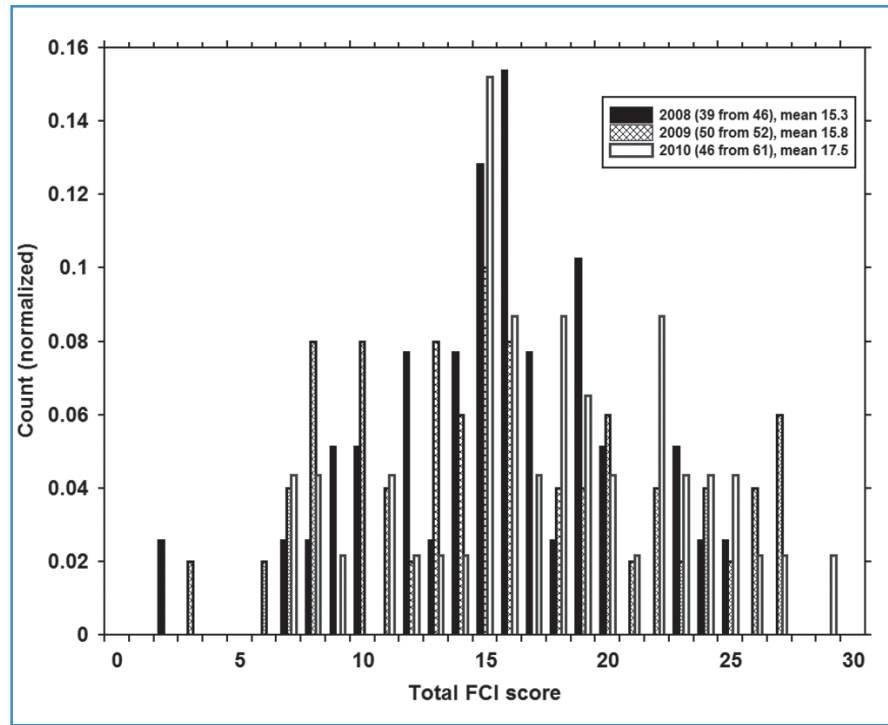

Figure 1. The incidence of total $\mathrm{FCl}$ scores across the three cohorts from 2008, 2009 and 2010

\section{Results}

Figure 1 shows the range of scores from the $\mathrm{FCl}$ corresponding to the intakes in 2008, 2009 and 2010. In all years the most common score is typically around 15 , but there are scores as low as 2 in 2008 and as high as 29 in 2010. The mean score in 2010 is slightly higher than in both 2008 and 2009 and reasons for this are being sought within average A-level scores in this intake. Nonetheless, the range of results across all years is very similar and makes it difficult to decide what to teach and at what level. In all years there are clearly students who have a good understanding of mechanics principles whilst there are also a significant number of students who do not.

Despite this wide variation in scores across the class, the breakdown of responses question by question (figure 2) reveals that the classes behave in a very similar manner. Although there are differences, especially between the 2008 and 2010 cohorts with a slightly higher proportion of the latter cohort giving the correct answers, there are also striking similarities. Where the majority of students give the correct answer in one year the same happens in the other years. Likewise, where the majority of students appear to struggle the same is also true in other years.

Three questions stand out as producing anomalously low numbers of correct responses; 5,15 and 26. In fact, as judged by the 2009 and 2010 cohorts question 5 appears quite similar to question 13, but the very low number of correct responses in 2008 marks this question out. We therefore concentrate on these three questions in addition to questions 2 and 11 . These last two are answered correctly by about $40 \%$ of the class, and as such do not stand out especially, but we focus on them for different reasons. Question 2 is interesting because it relates directly to question 1 , which around $80 \%$ of the class answer correctly. Question 11 indicates a particular alternative conception held by a significant proportion of the class.

Detailed analysis of these five questions, 2,5,11, 15 and 26, shows that not only do the different cohorts behave similarly when choosing the correct response, but also when choosing the incorrect responses.

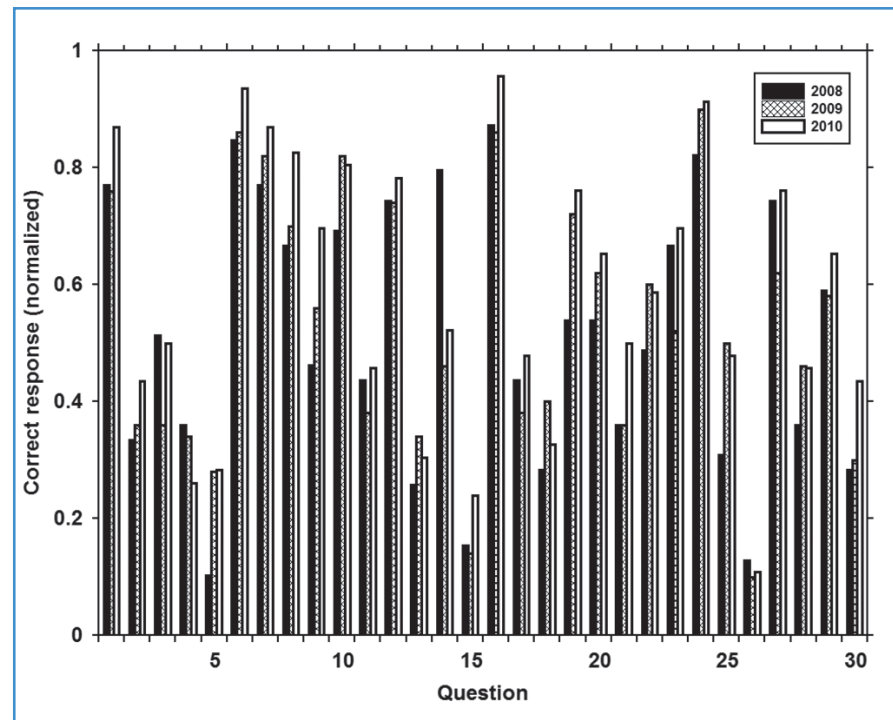

Figure 2: The breakdown of correct responses for each question. Though differences are apparent the trends are remarkably similar 


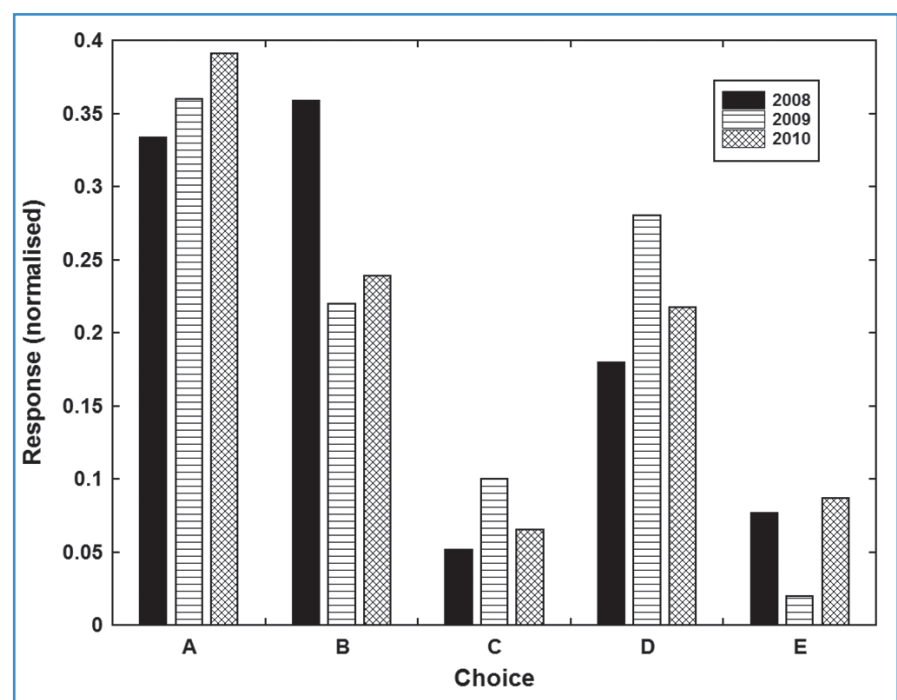

Figure 3: The breakdown of the different responses for question 2

Figure 3 shows the breakdown of the choices for question 2, which can only be understood properly in relation to question 1 , which is essentially about the famous experiment at the leaning tower of Pisa. Galileo's is reputed to have dropped two objects of different weights and observed the time taken to reach the ground, but in question 1 the objects are metal spheres, one weighing twice as much as the other, dropped from the roof of a single story building. Students are asked to choose from five possible semi-quantitative answers; for example, does the light ball take twice as long as the heavy ball to reach the ground, half as long, the same amount of time, or some other variation? Question 2 takes matters further and asks if the same two objects were to roll off a horizontal table with the same speed as each other, would the heavier ball land twice as close to the table as the lighter ball, twice as far away, the same distance away, or some other variation? Approximately half as many students as answer question 1 correctly also answer this question correctly (A), but interestingly the majority of incorrect answers in all years have the heavier ball landing closer to the table; half as close in $\mathrm{B}$ but considerably closer in $\mathrm{D}$. The options in which the lighter ball lands closer to the table ( $C$ and $E$ ) are chosen only by a small minority in each year.

The $\mathrm{FCl}$ doesn't reveal why students chose particular answers, and for question 1 it is not clear whether students are simply aware of the historical association with Galileo and therefore know the answer or whether they have reasoned out that the two balls must hit the ground at the same time because they are subject to the same acceleration. Likewise, it is not clear whether those who have answered question 2 correctly have reasoned out the answer or simply know it. The interesting fact is that there is a very large difference between the numbers answering the two questions correctly and the question arises as to why students are unable to reason out the answer. Two possibilities exist. First, it is evident from question 1 that the great majority of students should recognise that the two balls take exactly the same time to reach the floor, but students' knowledge is known to be context dependent ${ }^{11}$ and there is also evidence that students can hold conflicting views simultaneously ${ }^{12}$. It is possible, therefore, that changing the context from a roof top from which the balls are simply dropped to a table top from which the balls are

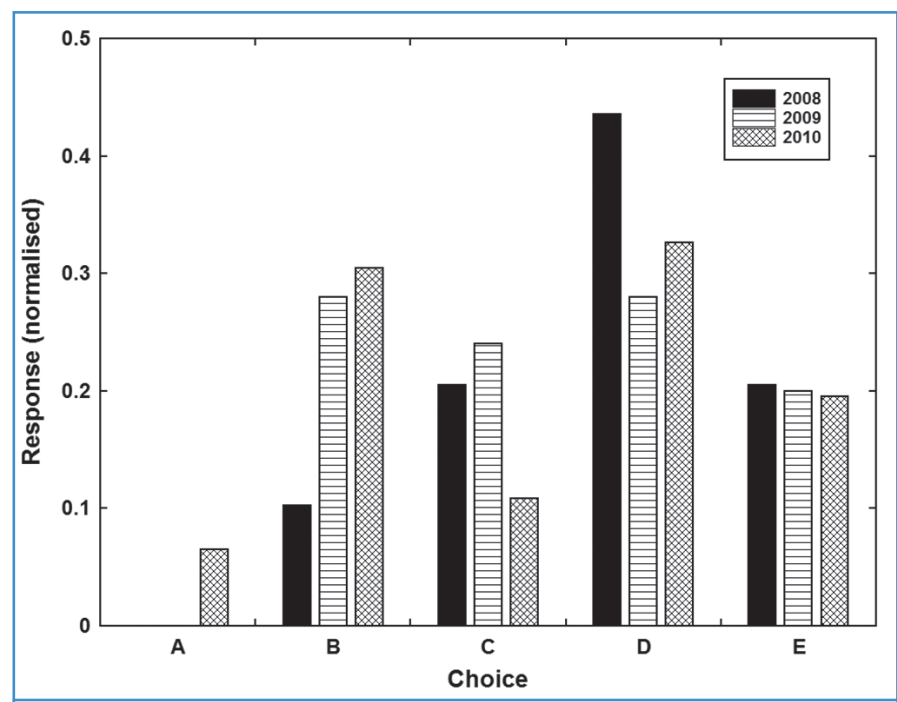

Figure 4: The breakdown of the different responses for question 5

launched with a horizontal velocity could lead students to fail to recognize that the flight times are equal. The second possibility is that the students know that the flight times are equal, as demonstrated in question 1 , but that a significant number are unable to apply this knowledge and reason out that, as the horizontal velocity remains unaffected by the acceleration due to gravity, the identical horizontal distances travelled must also be equal.

Figure 4 shows the breakdown of choices for question 5, with again strong similarities among the different years. In this question students are presented with the scenario of a frictionless channel with essentially a semi-circular profile placed on a horizontal table top. A ball enters the channel at a point $p$ and exits at a point $r$ having moved just over half the circumference of the circle. Students are asked to identify which of four forces are acting on the ball whilst it is at a point $q$ in the channel; a downward force of gravity, a force exerted by the channel pointing from $q$ towards the centre of the circle, an opposite force pointing from the centre to $q$, and a force in the direction of motion. The last two do not exist and the correct combination comprises the first two forces only (choice $B)$. Choice A identifies only the force of gravity and was not chosen by any students in 2008 and 2009, and only a handful of students in 2010. Choices C, D, and E, which collectively make up some $60-70 \%$ of the class, all identify among the combinations a force pointing in the direction of motion. As described, this a well known alternative conception about force. 


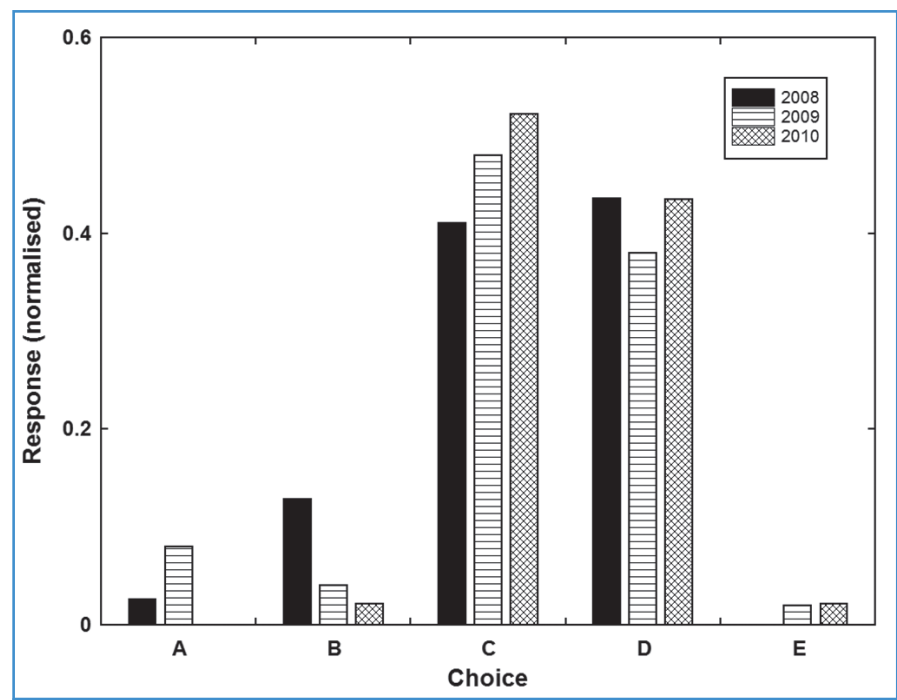

Figure 5: The breakdown of the different responses for question 11

There are differences between the years, but those differences are also revealing. For example, in the 2008 cohort the number choosing $B$ is significantly smaller than in 2009 and 2010, but interestingly those people appear to have chose $D$ instead. $B$ and $D$ are the only two options that identify a force acting from the ball to the centre, so it is consistently the case over the three years tested that only $30 \%$ of students can correctly identify this force as acting in this system. Likewise in 2010 there is a group of students who appear to have chosen $\mathrm{A}$ over $\mathrm{C}$ compared with the other two cohorts. However, these two are the only two options that do not identify any force between the ball and the centre and so again the proportion of the class who do not recognise such a forced is consistent from year to year at around $20 \%$. A similar number in each of the years have chosen $E$ and would therefore appear to believe that a force points from the centre to the ball. The numbers choosing these different incorrect answers might not in themselves be significant, but the consistency from year to year stands out.

Figure 5 shows the breakdown for question 11. As with question 5 , students are presented with a body moving horizontally along a frictionless path and are asked to identify the forces acting on it. In this case the body is a hockey puck which has been kicked and is now moving freely. The only forces acting on it therefore comprise the downward force of gravity and an upward reaction. Choices $C$ and $D$ are the only two that identify the upward reaction; choice $D$ correctly identifies only these two, but choice $C$ identifies both of these forces and a force in the direction of motion. Over $80 \%$ of the class would appear to recognise the existence of the reaction force, but approximately $55 \%$ would appear to believe also in a force in the direction of motion. This is fewer than the number who identify a similar force in question 5 , but otherwise supports the existence of this alternative conception among the majority of students. For completeness, other combinations comprise the downward force of gravity only (A), gravity and a force in the direction of motion (B), and no forces at all $(E)$.

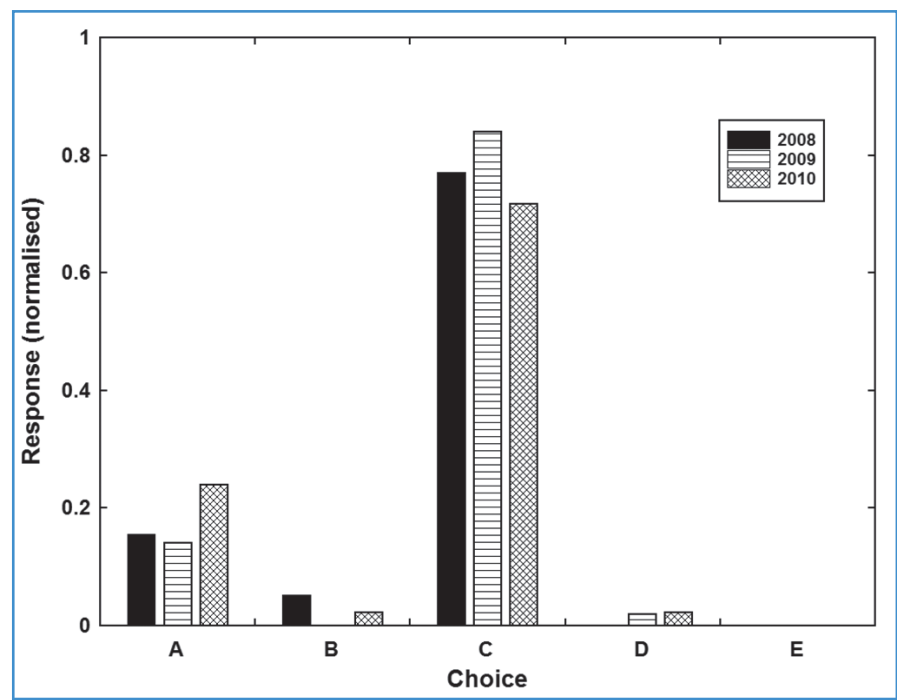

Figure 6: The breakdown of the different responses for question 15

Question 15 (figure 6) is related to question 16, which around $90 \%$ of students answered correctly. The concept being tested here is Newton's third law of motion and involves a car pushing on a truck. Students are asked to identify in both questions the magnitude of the force the truck exerts on the car in relation to the force the car exerts on the truck. However, in question 15 the car is accelerating whilst in question 16 the car is moving at a constant velocity. In all years the overwhelming choice in question 15 is $\mathrm{C}$, the car exerts a greater force on the truck than the truck exerts on the car, whilst in question 16 the forces are correctly identified as being equal in magnitude. In a number of cases the correct response to 15, A, was crossed out and $\mathrm{C}$ selected instead, which indicates that these students at least considered that the forces should be equal but were perhaps confused by the fact that the car is accelerating. Newton's second law identifies acceleration with a nett force and the great majority of students have opted for this. The responses to question 16 should also be considered in this light. On the face of it students would appear to have applied the third law, but the lack of acceleration might have led students to apply, albeit incorrectly, the second law instead and conclude that as the nett force must be zero, so the force exerted by the truck matches that exerted by the car. 


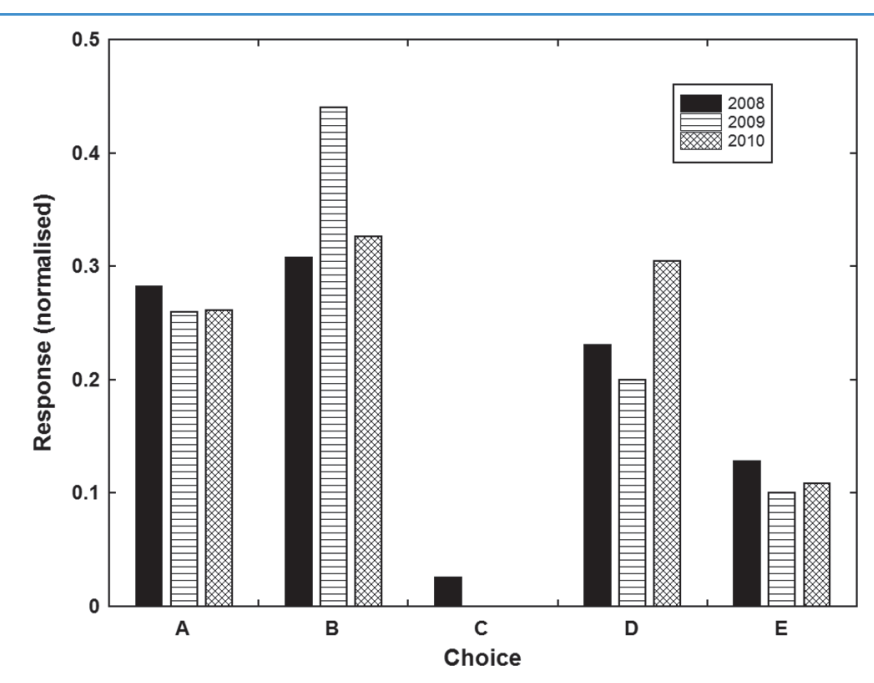

Figure 7: The breakdown of the different responses for question 26

Question 26 relates to Newton's second law, and as with so many questions in the $\mathrm{FCl}$ it relates to a situation set up in a previous question. In question 25 students are asked about the magnitude of a force with which a box is pushed across the floor at constant speed. Only $40-50 \%$ of students answered correctly that the force is equal in magnitude to the force resisting the motion of the box. Given the apparent confusion between Newton's second and third laws apparent in questions 15 and 16 and that over $80 \%$ answered question 16 correctly, this is perhaps a little surprising. In question 26 the force on the box is doubled and students are asked about the motion of the box. Newton's second law requires the box to accelerate (answer E), but as figure 7 shows, answers are split fairly evenly between $A, B$ and $D$, all of which express some variation on the idea that the speed increases initially but then remains constant. Students are probably guided by their own experience on this question. The question states that the box is pushed by a person and it is easy to imagine pushing a box at different speeds across a floor. Intuitively we might expect that different forces are being applied in each case. It is not really surprising, therefore, that these three options were so popular whilst $C$ and $E$, both of which involve increasing speeds, were either not chosen or chosen only by a minority. The key to this question lies both in understanding the preceding question and in reading the wording very carefully. Whilst experience might suggest that a box can be pushed across a floor at different speeds, Newton's second law implies that the applied force cannot be constant unless it is equal in magnitude to the resistive force opposing the motion. Indeed, the force applied to the box will vary as first one foot and then the other pushes against the ground and it could be argued that the question is unphysical in the sense that a person cannot generate a constant force over any extended distance. However, the question states that the applied force is doubled and by Newton's second law the box should accelerate.

\section{Conclusion}

There is an enormous volume of literature related to the $\mathrm{FCl}$ but the author is not aware of a similar analysis of the responses question by question and certainly nothing of this kind in relation to UK students. The present analysis reveals remarkable similarities over the three cohorts, implying that though there might be no such thing as a typical student there is at least a typical cohort characterised by a distribution of correct answers on the FCl. This similarity extends also to the choice of incorrect answers and might well indicate something systematic about the structure of mechanics knowledge at this level. Further analysis is required before such a conclusion can be drawn, but it seems to the author to be quite remarkable that three entirely separate cohorts drawn from different schools around the UK should all demonstrate such a similar structure in their collective knowledge.

Analysis of the $\mathrm{FCl}$ scores has also revealed a very large range of capabilities within a cohort, from barely any understanding of mechanics concepts right through to what amounts to a functional understanding. Hestenes has suggested $^{8}$ that a total $\mathrm{FCl}$ score of $18-20$ is the entry threshold to Newtonian thinking; below this score students do not use Newtonian concepts coherently in their thinking. By contrast score of 25 represents the threshold for mastery of Newtonian concepts. Among each cohort there is a group of students who exceeded the first threshold and a small number in both 2009 and 2010 who exceeded the second. According to this criterion the majority of students entering our first year are not Newtonian thinkers and this is reflected in their choice of incorrect answers. These include the idea that a force exists in the direction of motion as well as confusion between the second and third laws and in particular the idea that there can be a nett force acting even when equal and opposite reactive forces within the system are present. In addition, the analysis of question 2 points to an inability to reason qualitatively, either through being unable to recognise pertinent knowledge demonstrated in the previous question or to apply such knowledge.

Having thus identified the prior knowledge characteristic of A-level students entering university, the question then arises as to how to teach accordingly. The ineffectiveness of the traditional lecture in bringing about conceptual change has long been recognised within the physics education research community, largely because students are passive spectators, but within many UK HE institutions the lecture is still the predominant form of instruction ${ }^{14}$. If figure 2 is typical of students entering degree courses in other UK institutions in which the conventional lecture is still favoured there is good reason to suppose that for many such students conceptual development will be slow. Ideally students should be active and Hestenes has long advocated a role for modelling within the curriculum ${ }^{13}$. Modelling in this context means more than setting down mathematical equations and working through to a solution. In Hestenes view this is only one element of a model and in addition to the mathematics students need to be able to identify the different components of both the system and the environment, as well as their properties and interactions. Having developed a model students also need to be able to apply it to other situations in order to consolidate their knowledge. These aspects of modelling need to be taught explicitly, as, according to Hestenes ${ }^{14}$, "Much of it [modelling theory] is so basic and well known to physicists that they take it for granted and fail to realize that it should be taught to students". 
There may be other ways of actively engaging students, but this kind of modelling approach resonates with recent work by Nersessian on the construction of scientific concepts ${ }^{15}$ through what she calls "model-based reasoning". This is the ability to reason qualitatively through the use of various forms of representations and mental models. Although Nersessian was writing about the construction of concepts in the context of research and the development of scientific knowledge, there is no reason why the ideas are not applicable to the construction of concepts in the class room. However, students need to be taught explicitly the value of representing a problem by diagrams, equations, or even just words ${ }^{16}$. The present work has shown that students entering onto a UK physics degree are not in general Newtonian thinkers and that such active methods of instruction might be therefore needed within the UK. A first year mechanics course is as good a place as any to try to start teaching these skills.

\section{References}

1. Ausubel D. P. (1968): Educational psychology: A cognitive view. New York: Holt, Rinehart and Winston

2. di Sessa, Andrea (1987): The Third Revolution in Computers and Education, J. Res. Sci. Teach. 24(4) 343-367

3. Redish, Edward F. (2003): Teaching Physics With The Physics Suite, John Wiley \& Sons, USA.

4. Smith, Julia I. and Tanner, Kimberly (2010): The Problem Of Revealing How Students Think: Concept Inventories and Beyond, CBE-Life Science Education 9, 1-5

5. Wallace, Colin S. and Bailey, Janelle M. (2010): Do Concept Inventories Actually Measure Anything? Astronomy Education Review, 9, 010116-1.

6. Planinic, Maja; Ivanjek, Lana and Susac, Ana (2010): Rasch Model based analysis of the Force Concept Inventory, Physical Review Special Topics - Physics Education Research, 6, 010103

7. Huffman, Douglas and Heller, Patricia (1995): What Does the Force Concept Inventory Actually Measure? The Physics Teacher, 33, 138-143.

8. Hestenes, David and Halloun, Ibrahim ((1995): Interpreting The Force Concept Inventory: A Reply to Hestenes and Halloun, The Physics Teacher, 33, 502-506.

9. Heller, Patricia and Huffman, Douglas (1995): Interpreting The Force Concept Inventory: A response to Huffman and Heller, The Physics Teacher, 33, 503-511.

10. Sands, David (2010): First Year Mechanics Taught Through Modelling In Vpython, New Directions in the Teaching of Physical Sciences, Journal of the UK Physical Sciences Centre, University of Hull, Issue 6, 47-50.

11. Stewart, John; Griffin, Heather and Stewart, Gay (2007): Context Sensitivity in the Force Concept Inventory, Physical Review Special Topics - Physics Education Research, 3, 010102

12. Bao, Lei and Redish, Edward F. (2006): Model Analysis: Representing And Assessing The Dynamics Of Student Learning, Physical Review Special Topics - Physics Education Research, 2, 010103

13. McDermott, L.C. (2001): Oersted Medal Lecture 2001: Physics education research: The key to student learning, Am.J. Phys. 69 (11) 1127.

14. Hestenes, David (1987): Am. J. Phys, 55(5) 440-454

15. Nersessian, Nancy J. (2008) : Creating Scientific Concepts, Bradford books
16. Sands, David and Overton, Tina (2010): Cognitive psychology and problem solving in the physical sciences, New Directions in the Teaching of Physical Sciences, Issue 6 September 2010, Journal of the UK Physical Sciences Centre, University of Hull, p21-25 